CRYSTALLOGRAPHIC COMMUNICATIONS

ISSN 2056-9890

Received 27 March 2016

Accepted 27 March 2016

Edited by W. T. A. Harrison, University of Aberdeen, Scotland

Keywords: crystal structure; piperazine derivative; molecular salt; hydrogen bonding; Hirshfeld surface analysis.

CCDC reference: 1470800

Supporting information: this article has supporting information at journals.iucr.org/e

\section{trans-2,5-Dimethylpiperazine-1,4-diium bis(perchlorate) dihydrate: crystal structure and Hirshfeld surface analysis}

\author{
Cherifa Ben Mleh, ${ }^{a *}$ Thierry Roisnel $^{\mathrm{b}}$ and Houda Marouani ${ }^{\mathrm{a}}$
}

aLaboratoire de Chimie des Matériaux, Faculté des Sciences de Bizerte, 7021 Zarzouna Bizerte, Université de Carthage, Tunisia, and ${ }^{\mathbf{b}}$ Centre de Diffractométrie X, UMR 6226 CNRS, Unité Sciences Chimiques de Rennes, Université de Rennes I, 263 Avenue du, Général Leclerc, 35042 Rennes, France. *Correspondence e-mail: cherifa_benmleh@hotmail.com

The asymmetric unit of the title hydrated molecular salt, $\mathrm{C}_{6} \mathrm{H}_{16} \mathrm{~N}_{2}{ }^{2+}$.$2 \mathrm{ClO}_{4}{ }^{-} \cdot 2 \mathrm{H}_{2} \mathrm{O}$, contains a half dication (completed by inversion symmetry), a perchlorate anion and a water molecule. The extended structure consists of infinite chains of formula $\left[\left(\mathrm{ClO}_{4}\right) \mathrm{H}_{2} \mathrm{O}\right]_{n}{ }^{n-}$ ions extending along the $b$ axis linked by $\mathrm{O}_{\mathrm{w}}-\mathrm{H} \cdots \mathrm{O}(\mathrm{w}=$ water $)$ hydrogen bonds. These chains are cross-linked by the dications via $\mathrm{N}-\mathrm{H} \cdots \mathrm{O}_{\mathrm{w}}$ and weak $\mathrm{C}-\mathrm{H} \cdots \mathrm{O}$ hydrogen bonds, thus forming a three-dimensional supramolecular network. Three-dimensional Hirshfeld surface analysis and two-dimensional fingerprint maps reveal that the structure is dominated by $\mathrm{H} \cdots \mathrm{O} / \mathrm{O} \cdots \mathrm{H}$ and $\mathrm{H} \cdot \cdots \mathrm{H}$ contacts.

\section{Chemical context}

Piperazine $\left(\mathrm{C}_{4} \mathrm{H}_{10} \mathrm{~N}_{2}\right)$ and its derivatives are a family of strongly basic amines able to form dications, in which all of the $\mathrm{N}-\mathrm{H}$ bonds are generally active in hydrogen-bond formation. They are used in pharmacology and found in biologically active compounds across a number of different therapeutic areas, displaying antibacterial, antifungal, antimalarial, antipsychotic, antidepressant and antitumor activity (Brockunier et al., 2004; Bogatcheva et al., 2006).

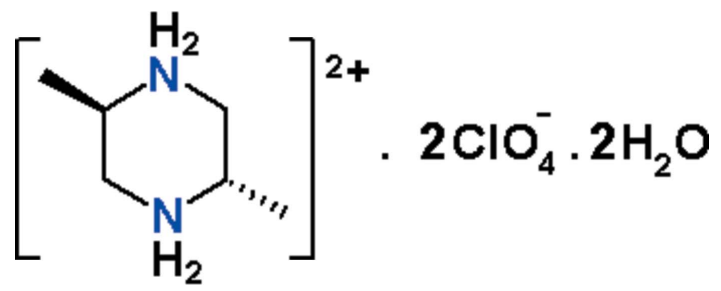

In this work, as part of our studies in this area, we report the preparation and structural investigation of a new hydrated perchlorate salt, $\mathrm{C}_{6} \mathrm{H}_{16} \mathrm{~N}_{2}{ }^{2+} \cdot 2 \mathrm{ClO}_{4}{ }^{-} \cdot 2 \mathrm{H}_{2} \mathrm{O}(\mathrm{I})$.

\section{Structural commentary}

The asymmetric unit of (I) is composed of a half of a trans-2,5dimethylpipeazine-1,4-dium dication, one perchlorate anion and one water molecule (Fig. 1). The complete dication is generated by crystallographic inversion symmetry, leading to a typical chair conformation, with the methyl groups occupying equatorial positions [puckering parameters: $Q=0.7341 \AA, \theta=$ 90 and $\varphi=-16^{\circ}$, which is similar the conformation of the same species in its nitrate salt (Gatfaoui et al., 2014). Other- 


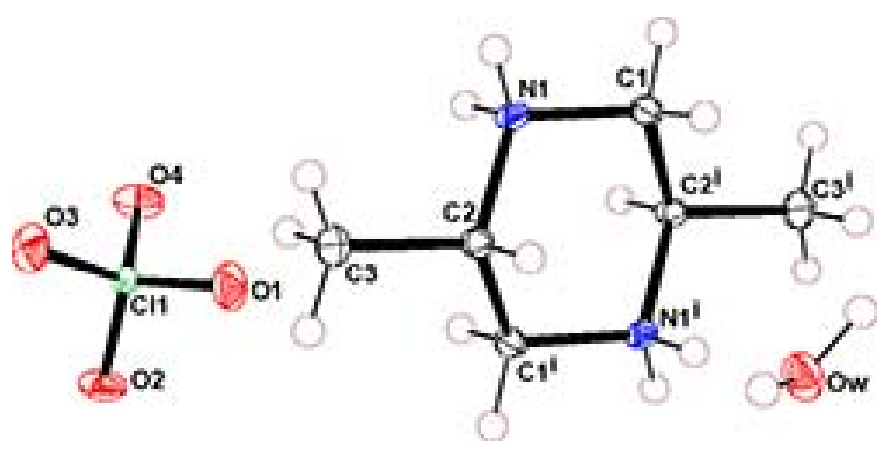

Figure 1

An ORTEP view of (I) with displacement ellipsoids drawn at the $30 \%$ probability level. Symmetry code: (i) $-x+\frac{1}{2},-y+\frac{1}{2},-z$.

wise, the bond lengths and angle in the dication are normal (Rother et al., 1997; Gatfaoui et al., 2014; Essid et al., 2015).

The perchlorate anion displays its expected tetrahedral geometry around the chlorine atom. Interatomic bond lengths and angles of the perchlorate anion lie respectively within the ranges [1.4327 (10)-1.4452 (11) ̊] and [109.01 (7)- 110.28 (7) ']. Similar geometrical features have also been noticed in other crystal structures (Toumi Akriche et al., 2010; Berrah et al., 2012).

\section{Supramolecular features}

In the extended structure, the anions are connected to the water molecules through $\mathrm{O}_{\mathrm{w}}-\mathrm{H} \cdots \mathrm{O}$ hydrogen bonds (Table 1), generating a corrugated $C_{2}^{2}(5)$ chain running along the [010] direction (Fig. 2). These chains are linked via the trans-2,5-dimethlpiperazine-1,4-diium cations through $\mathrm{N}-$ $\mathrm{H} \cdots \mathrm{O}, \mathrm{N}-\mathrm{H} \cdots \mathrm{O}_{\mathrm{w}}$ and weak $\mathrm{C}-\mathrm{H} \cdots \mathrm{O}$ hydrogen bonds,

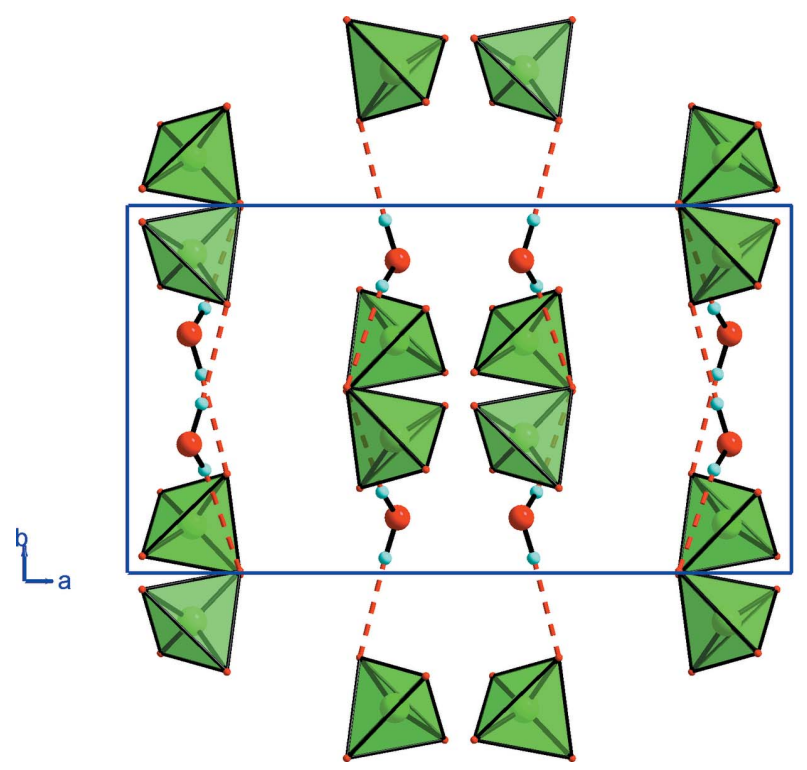

Figure 2

Hydrogen-bonded supramolecular chains involving anions and water molecules of compound (I), represented through the $a b$ plane.
Table 1

Hydrogen-bond geometry $\left(\AA{ }^{\circ}\right)$.

\begin{tabular}{lllll}
\hline$D-\mathrm{H} \cdots A$ & $D-\mathrm{H}$ & $\mathrm{H} \cdots A$ & $D \cdots A$ & $D-\mathrm{H} \cdots A$ \\
\hline $\mathrm{O} W-\mathrm{H} 1 W \cdots \mathrm{O} 1^{\mathrm{i}}$ & $0.85(1)$ & $2.03(1)$ & $2.8637(16)$ & $167(2)$ \\
$\mathrm{O} W-\mathrm{H} 2 W \cdots \mathrm{O} 2^{\mathrm{ii}}$ & $0.85(1)$ & $2.23(1)$ & $2.9932(16)$ & $150(2)$ \\
$\mathrm{N} 1-\mathrm{H} 1 N \cdots 4^{\text {iii }}$ & 0.90 & 2.18 & $2.9067(15)$ & 137 \\
$\mathrm{~N} 1-\mathrm{H} 1 N \cdots 3^{\text {iv }}$ & 0.90 & 2.42 & $3.0293(15)$ & 125 \\
$\mathrm{~N} 1-\mathrm{H} 1 N \cdots W^{\mathrm{v}}$ & 0.90 & 2.55 & $3.1994(16)$ & 130 \\
$\mathrm{~N} 1-\mathrm{H} 2 N \cdots W^{\mathrm{i}}$ & 0.90 & 1.91 & $2.8019(15)$ & 172 \\
$\mathrm{C} 1-\mathrm{H} 1 B \cdots \mathrm{O}^{\text {iv }}$ & 0.97 & 2.56 & $3.1007(17)$ & 116 \\
\hline
\end{tabular}

Symmetry codes: (i) $-x+\frac{1}{2},-y+\frac{3}{2},-z+1$; (ii) $-x+\frac{1}{2},-y+\frac{1}{2},-z+1$; (iii) $x,-y+1, z+\frac{1}{2}$; (iv) $-x, y+1,-z+\frac{1}{2}$; (v) $x-\frac{1}{2},-y+\frac{3}{2}, z-\frac{1}{2}$.

forming a three-dimensional supramolecular network (Fig. 3). These data show that each organic cation is connected to six inorganic chains.

\section{Hirshfeld surface analysis}

The three-dimensional Hirshfeld surfaces and two-dimensional fingerprint plots of (I) were prepared using CrystalExplorer (Wolff et al., 2012) and are shown in Figs. 4 and 5, respectively. The interaction between $\mathrm{N}-\mathrm{H}$ and oxygen atoms can be seen in the Hirshfeld surface as the bright-red area in Fig. 4 (labeled $a$ ). The light-red spots are due to $\mathrm{O}_{\mathrm{w}}-\mathrm{H} \cdots \mathrm{O}$ interactions (labeled $b$ ). For the salt, $\mathrm{O} \cdots \mathrm{H} / \mathrm{H} \cdots \mathrm{O}$ contacts, which are attributed to $\mathrm{N}-\mathrm{H} \cdots \mathrm{O}_{\mathrm{w}}$ and $\mathrm{O}_{\mathrm{w}}-\mathrm{H} \cdots \mathrm{O}$ hydrogen-bonding interactions, appear as two sharp symmetric spikes in the two-dimensional fingerprint maps. They have the most significant contribution to the total Hirshfeld surfaces. The $\mathrm{H} \cdot \cdot \mathrm{H}$ contacts appear in the middle of the scattered points in the two-dimensional fingerprint maps. For further information on Hirshfeld surfaces, see: Spackman \& McKinnon (2002) and Spackman \& Jayatilaka (2009).

\section{Synthesis and crystallization}

The title compound was prepared from an alcoholic solution containing trans-2,5-dimethylpiparazine ( $0.1 \mathrm{~g}, 1 \mathrm{mmol}$, purity

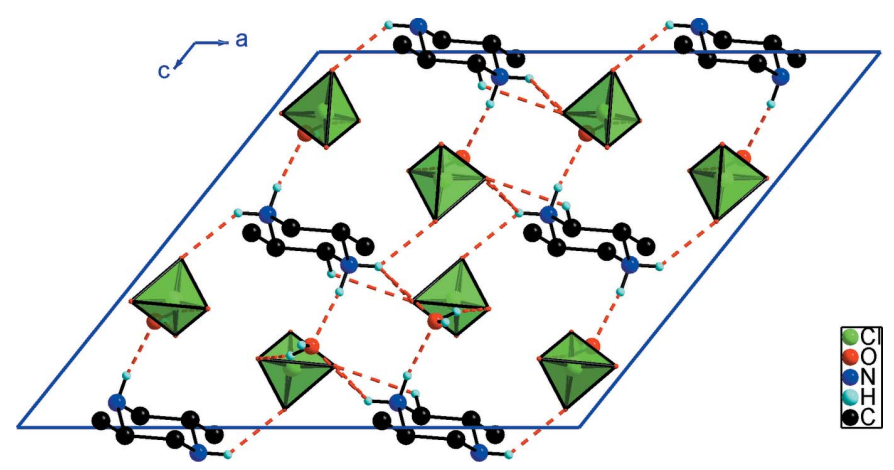

Figure 3

Projection of (I) along the $b$ axis. The H-atoms not involved in hydrogen bonding are omitted. 


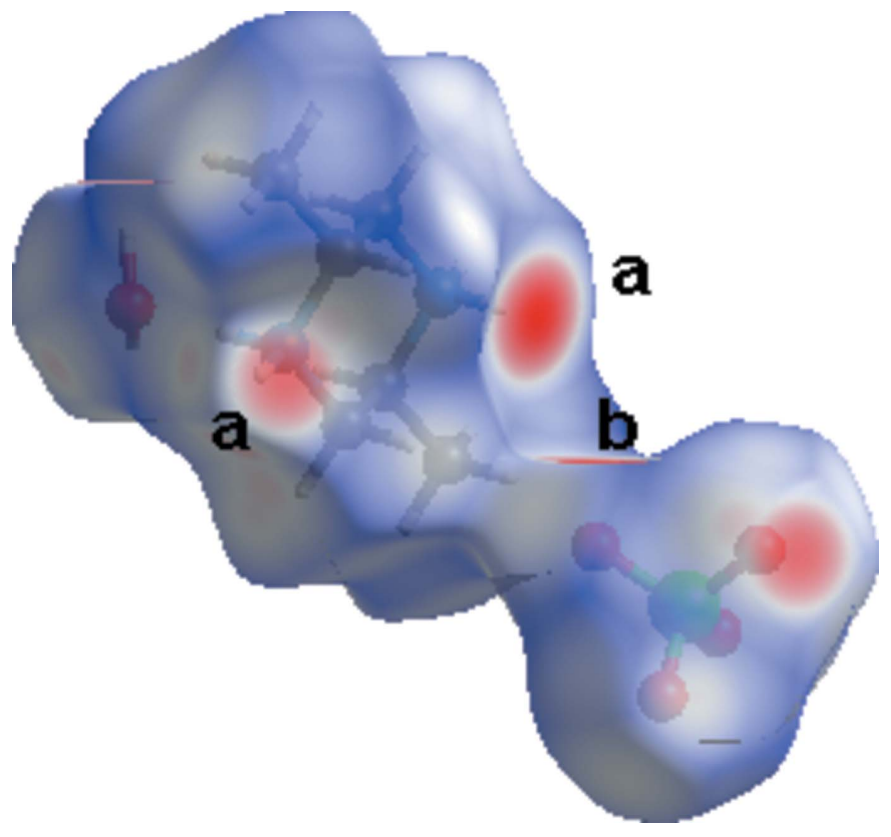

Figure 4

Hirshfeld surface around the constituents of (I) coloured according to $d_{\text {norm}}$. The surfaces are shown as transparent to allow visualization of the orientation and conformation of the functional groups.

(a)

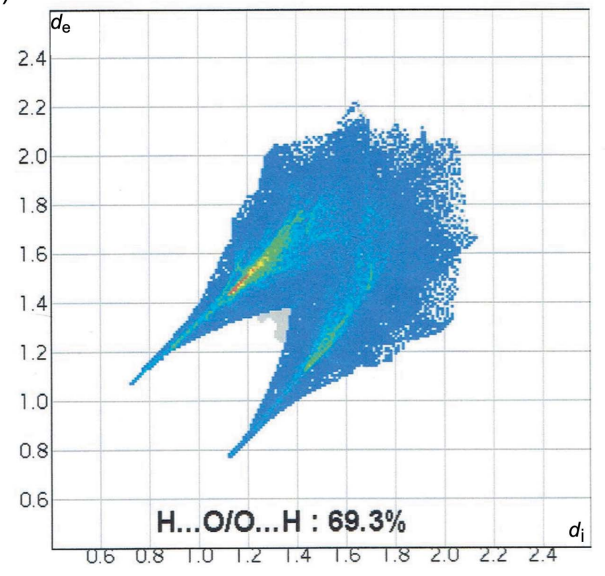

(b)

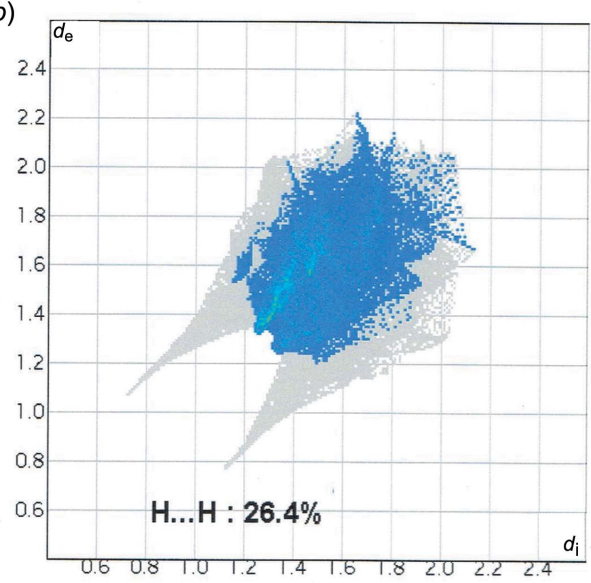

Figure 5

Fingerprint plots of the major contacts: $(a) \mathrm{H} \cdots \mathrm{O}$ and $(b) \mathrm{H} \cdots \mathrm{H}$.
Table 2

Experimental details.

Crystal data

Chemical formula

$M_{\mathrm{r}}$

Crystal system, space group

Temperature (K)

$a, b, c(\AA)$

$\beta\left({ }^{\circ}\right)$

$V\left(\AA^{3}\right)$

$Z$

Radiation type

$\mu\left(\mathrm{mm}^{-1}\right)$

Crystal size $(\mathrm{mm})$

$\mathrm{C}_{6} \mathrm{H}_{16} \mathrm{~N}_{2}{ }^{2+} \cdot 2 \mathrm{ClO}_{4}{ }^{-} \cdot 2 \mathrm{H}_{2} \mathrm{O}$

351.14

Monoclinic, C2/c

150

16.8603 (8), 7.2655 (3), 14.4534 (6)

$128.751(1)$

$1380.78(10)$

Mo $K \alpha$

0.52

$0.44 \times 0.29 \times 0.25$

Data collection

Diffractometer

Absorption correction

$T_{\min }, T_{\max }$

No. of measured, independent and observed $[I>2 \sigma(I)]$ reflections

$R_{\text {int }}$
$(\sin \theta / \lambda)_{\max }\left(\AA^{-1}\right)$

Bruker D8 VENTURE

Multi-scan (SADABS; Bruker, 2014)

$0.775,0.878$

$7760,1557,1457$

0.023

0.649

Refinement

$R\left[F^{2}>2 \sigma\left(F^{2}\right)\right], w R\left(F^{2}\right), S$

No. of reflections

No. of parameters

No. of restraints

$\mathrm{H}$-atom treatment

$0.028,0.074,1.13$

1557

100

3

$\mathrm{H}$ atoms treated by a mixture of independent and constrained refinement

$\Delta \rho_{\max }, \Delta \rho_{\min }\left(\mathrm{e} \AA^{-3}\right)$ $0.34,-0.41$

Computer programs: APEX2 and SAINT (Bruker, 2014), SIR97 (Altomare et al., 1999), SHELXL2014/7 (Sheldrick, 2015), ORTEP-3 for Windows and WinGX publication routines (Farrugia, 2012).

99\%, Aldrich) dissolved in ethanol $(20 \mathrm{ml})$ and perchloric acid $\mathrm{HClO}_{4}(0.2 \mathrm{~g}, 2 \mathrm{mmol}$, purity $96 \%$, Aldrich) with a molar ratio of 1:2. This mixture was stirred for $1 \mathrm{~h}$. After a week of evaporation at room temperature, colorless single crystals of suitable dimensions for crystallographic study were formed, and were isolated by filtration and washed with a small amount of distilled water. The crystals can be stable for months under normal conditions of temperature and humidity.

\section{Refinement}

Crystal data, data collection and structure refinement details are summarized in Table 2. All $\mathrm{H}$ atoms were located in a difference map but were placed geometrically and refined using a riding model, with $\mathrm{C}-\mathrm{H}=0.96 \AA$ (methyl), or $0.98 \AA$ (methine), $\mathrm{N}-\mathrm{H}=0.90 \AA\left(\mathrm{NH}_{2}\right)$ with $U_{\text {iso }}(\mathrm{H})=1.2 U_{\text {eq }}(\mathrm{C}$ or $\mathrm{N})$. The $\mathrm{H}$ atoms of the water molecule were refined with a distance restraint of $\mathrm{O}-\mathrm{H}=0.85$ (1) $\AA$ using DFIX and DANG commands (Sheldrick, 2015) with $U_{\text {iso }}(\mathrm{H})=1.5 U_{\text {eq }}(\mathrm{O})$.

\section{Acknowledgements}

This work was supported by the Tunisian Ministry of Higher Education Scientific Research. 


\section{References}

Altomare, A., Burla, M. C., Camalli, M., Cascarano, G. L., Giacovazzo, C., Guagliardi, A., Moliterni, A. G. G., Polidori, G. \& Spagna, R. (1999). J. Appl. Cryst. 32, 115-119.

Berrah, F., Bouacida, S., Anana, H. \& Roisnel, T. (2012). Acta Cryst. E64, o1601-o1602.

Bogatcheva, E., Hanrahan, C., Nikonenko, B., Samala, R., Chen, P., Gearhart, J., Barbosa, F., Einck, L., Nacy, C. A. \& Protopopova, M. (2006). J. Med. Chem. 49, 3045-3048.

Brockunier, L. L., He, J., Colwell, L. F. Jr, Habulihaz, B., He, H., Leiting, B., Lyons, K. A., Marsilio, F., Patel, R. A., Teffera, Y., Wu, J. K., Thornberry, N. A., Weber, A. E. \& Parmee, E. R. (2004). Bioorg. Med. Chem. Lett. 14, 4763-4766.

Bruker (2014). APEX2, SAINT and SADABS. Bruker AXS Inc., Madison, Wisconsin, USA.
Essid, M., Roisnel, T., Rzaigui, M. \& Marouani, H. (2015). Monatsh. Chem. DOI 10.1007/s00706-015-1485-9

Farrugia, L. J. (2012). J. Appl. Cryst. 45, 849-854.

Gatfaoui, S., Roisnel, T., Dhaouadi, H. \& Marouani, H. (2014). Acta Cryst. E70, o725.

Rother, G., Worzala, H. \& Bentrup, U. (1997). Z. Kristallogr. New Cryst. Struct. 212, 199.

Sheldrick, G. M. (2015). Acta Cryst. C71, 3-8.

Spackman, M. A. \& Jayatilaka, D. (2009). CrystEngComm, 11, 19-32. Spackman, M. A. \& McKinnon, J. J. (2002). CrystEngComm, 4, 378392.

Toumi Akriche, S., Rzaigui, M., Al-Hokbany, N. \& Mahfouz, R. M. (2010). Acta Cryst. E66, o300.

Wolff, S. K., Grimwood, D. J., McKinnon, J. J., Turner, M. J., Jayatilaka, D. \& Spackman, M. A. (2012). CrystalExplorer. University of Western Australia. 


\section{supporting information}

Acta Cryst. (2016). E72, 593-596 [https://doi.org/10.1107/S205698901600520X]

trans-2,5-Dimethylpiperazine-1,4-diium bis(perchlorate) dihydrate: crystal structure and Hirshfeld surface analysis

\section{Cherifa Ben Mleh, Thierry Roisnel and Houda Marouani}

Computing details

Data collection: APEX2 (Bruker, 2014); cell refinement: SAINT (Bruker, 2014); data reduction: SAINT (Bruker, 2014); program(s) used to solve structure: SIR97 (Altomare et al., 1999); program(s) used to refine structure: SHELXL2014/7 (Sheldrick, 2015); molecular graphics: ORTEP-3 for Windows (Farrugia, 2012); software used to prepare material for publication: WinGX publication routines (Farrugia, 2012).

trans-2,5-Dimethylpiperazine-1,4-diium bis(perchlorate) dihydrate

Crystal data

$\mathrm{C}_{6} \mathrm{H}_{16} \mathrm{~N}_{2}{ }^{2+} \cdot 2 \mathrm{ClO}_{4}^{-} \cdot 2 \mathrm{H}_{2} \mathrm{O}$

$M_{r}=351.14$

Monoclinic, $C 2 / c$

$a=16.8603(8) \AA$

$b=7.2655(3) \AA$

$c=14.4534(6) \AA$

$\beta=128.751(1)^{\circ}$

$V=1380.78(10) \AA^{3}$

$Z=4$

\section{Data collection}

D8 VENTURE Bruker AXS diffractometer

Radiation source: Incoatec microfocus sealed tube

Multilayer monochromator

rotation images scans

Absorption correction: multi-scan

(SADABS; Bruker, 2014)

$T_{\min }=0.775, T_{\max }=0.878$

Refinement

Refinement on $F^{2}$

Least-squares matrix: full

$R\left[F^{2}>2 \sigma\left(F^{2}\right)\right]=0.028$

$w R\left(F^{2}\right)=0.074$

$S=1.13$

1557 reflections

100 parameters

3 restraints
$F(000)=736$

$D_{\mathrm{x}}=1.689 \mathrm{Mg} \mathrm{m}^{-3}$

Mo $K \alpha$ radiation, $\lambda=0.71073 \AA$

Cell parameters from 7552 reflections

$\theta=3.1-27.5^{\circ}$

$\mu=0.52 \mathrm{~mm}^{-1}$

$T=150 \mathrm{~K}$

Prism, colourless

$0.44 \times 0.29 \times 0.25 \mathrm{~mm}$

7760 measured reflections

1557 independent reflections

1457 reflections with $I>2 \sigma(I)$

$R_{\text {int }}=0.023$

$\theta_{\text {max }}=27.5^{\circ}, \theta_{\min }=3.1^{\circ}$

$h=-21 \rightarrow 21$

$k=-9 \rightarrow 9$

$l=-18 \rightarrow 15$
Primary atom site location: structure-invariant direct methods

Secondary atom site location: difference Fourier map

Hydrogen site location: inferred from neighbouring sites

$\mathrm{H}$ atoms treated by a mixture of independent and constrained refinement 
$w=1 /\left[\sigma^{2}\left(F_{\mathrm{o}}^{2}\right)+(0.0308 P)^{2}+1.9533 P\right]$

where $P=\left(F_{\mathrm{o}}^{2}+2 F_{\mathrm{c}}^{2}\right) / 3$

$(\Delta / \sigma)_{\max }=0.001$

$$
\Delta \rho_{\max }=0.34 \text { e } \AA^{-3}
$$

\section{Special details}

Geometry. All esds (except the esd in the dihedral angle between two 1.s. planes) are estimated using the full covariance matrix. The cell esds are taken into account individually in the estimation of esds in distances, angles and torsion angles; correlations between esds in cell parameters are only used when they are defined by crystal symmetry. An approximate (isotropic) treatment of cell esds is used for estimating esds involving l.s. planes.

Refinement. Refinement of $\mathrm{F}^{2}$ against ALL reflections. The weighted R-factor $\mathrm{wR}$ and goodness of fit $\mathrm{S}$ are based on $\mathrm{F}^{2}$, conventional R-factors $R$ are based on $F$, with $F$ set to zero for negative $F^{2}$. The threshold expression of $F^{2}>2 \operatorname{sigma}\left(\mathrm{F}^{2}\right)$ is used only for calculating R-factors(gt) etc. and is not relevant to the choice of reflections for refinement. R-factors based on $\mathrm{F}^{2}$ are statistically about twice as large as those based on F, and R- factors based on ALL data will be even larger.

Fractional atomic coordinates and isotropic or equivalent isotropic displacement parameters $\left(\AA^{2}\right)$

\begin{tabular}{lllll}
\hline & $x$ & $y$ & $z$ & $U_{\text {iso }} / U_{\text {eq }}$ \\
\hline C11 & $0.09748(2)$ & $0.13197(4)$ & $0.16201(3)$ & $0.01195(12)$ \\
O1 & $0.14991(10)$ & $0.26958(16)$ & $0.25415(10)$ & $0.0307(3)$ \\
O2 & $0.16959(8)$ & $-0.00473(16)$ & $0.18348(11)$ & $0.0254(3)$ \\
O3 & $0.02214(9)$ & $0.04530(16)$ & $0.16384(10)$ & $0.0239(3)$ \\
O4 & $0.05035(9)$ & $0.21898(16)$ & $0.04934(9)$ & $0.0236(3)$ \\
OW & $0.40676(9)$ & $0.84994(14)$ & $0.78311(9)$ & $0.0203(2)$ \\
H1W & $0.3881(18)$ & $0.9600(15)$ & $0.780(2)$ & $0.046(7)^{*}$ \\
H2W & $0.3837(15)$ & $0.780(2)$ & $0.8086(18)$ & $0.034(6)^{*}$ \\
N1 & $0.14181(8)$ & $0.75580(15)$ & $0.43347(10)$ & $0.0111(2)$ \\
H2N & 0.1201 & 0.7208 & 0.3612 & $0.013^{*}$ \\
H1N & 0.0867 & 0.7774 & 0.4286 & $0.013^{*}$ \\
C1 & $0.20218(10)$ & $0.92935(18)$ & $0.46870(12)$ & $0.0119(3)$ \\
H1A & 0.2220 & 0.9720 & 0.5442 & $0.014^{*}$ \\
H1B & 0.1605 & 1.0239 & 0.4099 & $0.014^{*}$ \\
C2 & $0.20319(10)$ & $0.60230(18)$ & $0.52072(11)$ & $0.0114(3)$ \\
H2 & 0.2243 & 0.6396 & 0.5984 & $0.014^{*}$ \\
C3 & $0.13915(11)$ & $0.42938(19)$ & $0.48147(13)$ & $0.0186(3)$ \\
H3A & 0.0801 & 0.4542 & 0.4754 & $0.028^{*}$ \\
H3B & 0.1785 & 0.3334 & 0.5385 & $0.028^{*}$ \\
H3C & 0.1183 & 0.3911 & 0.4056 & $0.028^{*}$
\end{tabular}

Atomic displacement parameters $\left(\AA^{2}\right)$

\begin{tabular}{lllllll}
\hline & $U^{11}$ & $U^{22}$ & $U^{33}$ & $U^{12}$ & $U^{13}$ & $U^{23}$ \\
\hline C11 & $0.01181(18)$ & $0.01242(18)$ & $0.01379(18)$ & $-0.00022(10)$ & $0.00907(14)$ & $0.00166(10)$ \\
O1 & $0.0321(6)$ & $0.0200(6)$ & $0.0225(6)$ & $-0.0075(5)$ & $0.0085(5)$ & $-0.0069(5)$ \\
O2 & $0.0215(6)$ & $0.0256(6)$ & $0.0347(6)$ & $0.0119(5)$ & $0.0203(5)$ & $0.0101(5)$ \\
O3 & $0.0246(6)$ & $0.0246(6)$ & $0.0358(6)$ & $-0.0064(4)$ & $0.0253(5)$ & $-0.0012(5)$ \\
O4 & $0.0269(6)$ & $0.0308(6)$ & $0.0190(5)$ & $0.0105(5)$ & $0.0171(5)$ & $0.0119(4)$ \\
OW & $0.0272(6)$ & $0.0152(5)$ & $0.0174(5)$ & $-0.0013(4)$ & $0.0135(5)$ & $0.0000(4)$ \\
N1 & $0.0077(5)$ & $0.0131(5)$ & $0.0124(5)$ & $0.0006(4)$ & $0.0062(4)$ & $0.0008(4)$ \\
C1 & $0.0120(6)$ & $0.0101(6)$ & $0.0142(6)$ & $0.0011(5)$ & $0.0084(5)$ & $0.0006(5)$
\end{tabular}




\begin{tabular}{lllllll}
$\mathrm{C} 2$ & $0.0110(6)$ & $0.0113(6)$ & $0.0122(6)$ & $0.0008(5)$ & $0.0074(5)$ & $0.0020(5)$ \\
$\mathrm{C} 3$ & $0.0163(6)$ & $0.0139(6)$ & $0.0230(7)$ & $-0.0034(5)$ & $0.0111(6)$ & $0.0008(5)$ \\
\hline
\end{tabular}

Geometric parameters $\left(A,{ }^{\circ}\right)$

\begin{tabular}{|c|c|c|c|}
\hline $\mathrm{Cl} 1-\mathrm{O} 3$ & $1.4327(10)$ & $\mathrm{C} 1-\mathrm{C} 2^{\mathrm{i}}$ & $1.5218(17)$ \\
\hline $\mathrm{Cl1}-\mathrm{O} 4$ & $1.4363(10)$ & $\mathrm{C} 1-\mathrm{H} 1 \mathrm{~A}$ & 0.9700 \\
\hline $\mathrm{Cl1}-\mathrm{O} 1$ & $1.4425(11)$ & $\mathrm{C} 1-\mathrm{H} 1 \mathrm{~B}$ & 0.9700 \\
\hline $\mathrm{C} 11-\mathrm{O} 2$ & $1.4452(11)$ & $\mathrm{C} 2-\mathrm{C} 3$ & $1.5163(18)$ \\
\hline $\mathrm{OW}-\mathrm{H} 1 \mathrm{~W}$ & $0.850(9)$ & $\mathrm{C} 2-\mathrm{C}^{\mathrm{i}}$ & $1.5218(17)$ \\
\hline $\mathrm{OW}-\mathrm{H} 2 \mathrm{~W}$ & $0.850(9)$ & $\mathrm{C} 2-\mathrm{H} 2$ & 0.9800 \\
\hline $\mathrm{N} 1-\mathrm{C} 1$ & $1.4955(16)$ & $\mathrm{C} 3-\mathrm{H} 3 \mathrm{~A}$ & 0.9600 \\
\hline $\mathrm{N} 1-\mathrm{C} 2$ & $1.5071(16)$ & $\mathrm{C} 3-\mathrm{H} 3 \mathrm{~B}$ & 0.9600 \\
\hline $\mathrm{N} 1-\mathrm{H} 2 \mathrm{~N}$ & 0.9000 & $\mathrm{C} 3-\mathrm{H} 3 \mathrm{C}$ & 0.9600 \\
\hline $\mathrm{N} 1-\mathrm{H} 1 \mathrm{~N}$ & 0.9000 & & \\
\hline $\mathrm{O} 3-\mathrm{Cl1}-\mathrm{O} 4$ & $110.28(7)$ & $\mathrm{N} 1-\mathrm{C} 1-\mathrm{H} 1 \mathrm{~B}$ & 109.5 \\
\hline $\mathrm{O} 3-\mathrm{Cl1}-\mathrm{O} 1$ & $109.01(7)$ & $\mathrm{C} 2 \mathrm{i}-\mathrm{C} 1-\mathrm{H} 1 \mathrm{~B}$ & 109.5 \\
\hline $\mathrm{O} 4-\mathrm{Cl1}-\mathrm{O} 1$ & $109.03(7)$ & $\mathrm{H} 1 \mathrm{~A}-\mathrm{C} 1-\mathrm{H} 1 \mathrm{~B}$ & 108.1 \\
\hline $\mathrm{O} 3-\mathrm{Cl1}-\mathrm{O} 2$ & $109.29(7)$ & $\mathrm{N} 1-\mathrm{C} 2-\mathrm{C} 3$ & $110.17(10)$ \\
\hline $\mathrm{O} 4-\mathrm{C} 11-\mathrm{O} 2$ & $109.87(7)$ & $\mathrm{N} 1-\mathrm{C} 2-\mathrm{C} 1^{\mathrm{i}}$ & $108.88(10)$ \\
\hline $\mathrm{O} 1-\mathrm{C} 11-\mathrm{O} 2$ & $109.34(7)$ & $\mathrm{C} 3-\mathrm{C} 2-\mathrm{C} 1^{\mathrm{i}}$ & $111.63(11)$ \\
\hline $\mathrm{H} 1 \mathrm{~W}-\mathrm{OW}-\mathrm{H} 2 \mathrm{~W}$ & $109.1(17)$ & $\mathrm{N} 1-\mathrm{C} 2-\mathrm{H} 2$ & 108.7 \\
\hline $\mathrm{C} 1-\mathrm{N} 1-\mathrm{C} 2$ & $111.99(10)$ & $\mathrm{C} 3-\mathrm{C} 2-\mathrm{H} 2$ & 108.7 \\
\hline $\mathrm{C} 1-\mathrm{N} 1-\mathrm{H} 2 \mathrm{~N}$ & 109.2 & $\mathrm{C} 1{ }^{\mathrm{i}}-\mathrm{C} 2-\mathrm{H} 2$ & 108.7 \\
\hline $\mathrm{C} 2-\mathrm{N} 1-\mathrm{H} 2 \mathrm{~N}$ & 109.2 & $\mathrm{C} 2-\mathrm{C} 3-\mathrm{H} 3 \mathrm{~A}$ & 109.5 \\
\hline $\mathrm{C} 1-\mathrm{N} 1-\mathrm{H} 1 \mathrm{~N}$ & 109.2 & $\mathrm{C} 2-\mathrm{C} 3-\mathrm{H} 3 \mathrm{~B}$ & 109.5 \\
\hline $\mathrm{C} 2-\mathrm{N} 1-\mathrm{H} 1 \mathrm{~N}$ & 109.2 & $\mathrm{H} 3 \mathrm{~A}-\mathrm{C} 3-\mathrm{H} 3 \mathrm{~B}$ & 109.5 \\
\hline $\mathrm{H} 2 \mathrm{~N}-\mathrm{N} 1-\mathrm{H} 1 \mathrm{~N}$ & 107.9 & $\mathrm{C} 2-\mathrm{C} 3-\mathrm{H} 3 \mathrm{C}$ & 109.5 \\
\hline $\mathrm{N} 1-\mathrm{C} 1-\mathrm{C} 2^{\mathrm{i}}$ & $110.74(10)$ & $\mathrm{H} 3 \mathrm{~A}-\mathrm{C} 3-\mathrm{H} 3 \mathrm{C}$ & 109.5 \\
\hline $\mathrm{N} 1-\mathrm{C} 1-\mathrm{H} 1 \mathrm{~A}$ & 109.5 & $\mathrm{H} 3 \mathrm{~B}-\mathrm{C} 3-\mathrm{H} 3 \mathrm{C}$ & 109.5 \\
\hline $\mathrm{C} 22^{\mathrm{i}}-\mathrm{C} 1-\mathrm{H} 1 \mathrm{~A}$ & 109.5 & & \\
\hline
\end{tabular}

Symmetry code: (i) $-x+1 / 2,-y+3 / 2,-z+1$.

Hydrogen-bond geometry $\left(A,{ }^{\circ}\right)$

\begin{tabular}{lllll}
\hline$D-\mathrm{H} \cdots A$ & $D-\mathrm{H}$ & $\mathrm{H} \cdots A$ & $D \cdots A$ & $D-\mathrm{H} \cdots A$ \\
\hline $\mathrm{O} W-\mathrm{H} 1 W \cdots \mathrm{O} 1^{\mathrm{i}}$ & $0.85(1)$ & $2.03(1)$ & $2.8637(16)$ & $167(2)$ \\
$\mathrm{O} W-\mathrm{H} 2 W \cdots \mathrm{O} 2^{\text {ii }}$ & $0.85(1)$ & $2.23(1)$ & $2.9932(16)$ & $150(2)$ \\
$\mathrm{N} 1-\mathrm{H} 1 N \cdots \mathrm{O} 4^{\text {iii }}$ & 0.90 & 2.18 & $2.9067(15)$ & 137 \\
$\mathrm{~N} 1-\mathrm{H} 1 N \cdots \mathrm{O} 3^{\text {iv }}$ & 0.90 & 2.42 & $3.0293(15)$ & 125 \\
$\mathrm{~N} 1-\mathrm{H} 1 N \cdots \mathrm{O} W^{v}$ & 0.90 & 2.55 & $3.1994(16)$ & 130 \\
$\mathrm{~N} 1-\mathrm{H} 2 N \cdots \mathrm{O} W^{\text {i }}$ & 0.90 & 1.91 & $2.8019(15)$ & 172 \\
$\mathrm{C} 1-\mathrm{H} 1 B \cdots \mathrm{O} 3^{\text {iv }}$ & 0.97 & 2.56 & $3.1007(17)$ & 116 \\
\hline
\end{tabular}

Symmetry codes: (i) $-x+1 / 2,-y+3 / 2,-z+1$; (ii) $-x+1 / 2,-y+1 / 2,-z+1$; (iii) $x,-y+1, z+1 / 2$; (iv) $-x, y+1,-z+1 / 2$; (v) $x-1 / 2,-y+3 / 2, z-1 / 2$. 